\title{
A Strategy for High-Performance Photodetector based on Gra- phene-Si heterostructure
}

\author{
Tianying He, ${ }^{1,2}$ Changyong Lan, ${ }^{1,2, *}$ Chun Li, ${ }^{1,2,3}$ Sihan Zhou ${ }^{1,2}$, Yi Yin ${ }^{1,2}$ \\ 1, State Key Laboratory of Electronic Thin Films and Integrated Devices, University of Electronic Science and Technology of China, \\ Chengdu 610054, China \\ 2, School of Optoelectronic Science and Engineering, University of Electronic Science and Technology of China, Chengdu, 610054, China \\ 3, Wuhan National Laboratory for Optoelectronics, Huazhong University of Science and Technology, Wuhan 430074, China
}

\begin{abstract}
Van der Waals heterostructure shows promising applications in next generation optoelectronics. As a kind of van der Waals heterostructure, graphene/silicon $(\mathrm{Gr} / \mathrm{Si})$ based heterostructure devices have been demonstrated as high performance photodetectors. Here, we studied the origin of the high performance of $\mathrm{Gr} / \mathrm{Si}$ photodetectors based on photocurrent mapping technology. According to photocurrent mapping, the photocurrent in the $\mathrm{Gr} / \mathrm{Si}$ and $\mathrm{Gr} / \mathrm{SiO}_{2} / \mathrm{Si}$ area nearing the $\mathrm{Si}$ window edge is higher than that in other positions, which is attributed to the highly effective collection efficiency of photocarriers. A device with size of $\mathrm{Gr} / \mathrm{Si}$ region $(r=7.4 \mu \mathrm{m})$ and $\mathrm{Gr} / \mathrm{SiO}_{2} / \mathrm{Si}$ region $(L=6.1 \mu \mathrm{m})$ shows high sensitivity and a broadband photoresponse in the range from 420 to $1000 \mathrm{~nm}$ with the peak sensitivity of $52 \mathrm{~A} / \mathrm{W}$ at $780 \mathrm{~nm}$, and fast response speed with rise time of $16 \mu \mathrm{s}$ and decay time of $52 \mu \mathrm{s}$. Our study provides a strategy for the design of high photoresponsivity $\mathrm{Gr} / \mathrm{Si}$ based devices.
\end{abstract}

\section{INTRODUCTION}

Layered two-dimensional (2D) materials have attracted tremendous attention in recent years due to their excellent physical and chemical properties. Due to the passivated surface of layered 2D materials, it is easy to build 2D material based heterostructure by stacking layered 2D material on other kinds of materials without considering lattice mismatch [1]. Due to the mature processing technology of $\mathrm{Si}$, the study on 2D-Si heterostructure has become a hot topic in recent years $[2,3]$. Graphene(Gr)/Si heterostructure has excellent optoelectronic properties and shows promising applications in rectifies [4], sensors [5], solar cells [6], and photodetectors [2, 7-10]. The Gr/Si Schottky photodetectors have showed high performance photodetection from ultraviolet (UV) to near-infrared (NIR) [9-12] For example, Riazimehr et al. [12] achieved photodetection for wavelengths ranging from 380 to $1930 \mathrm{~nm}$. However, the responsivity of the photodetector is still limited due to the fast recombination of the photocarriers. Therefore, many strategies have been explored to enhance the performance of Gr/Si photodetectors. Riazimehr et al.[10] presented an interdigitated patterned $\mathrm{Gr} / \mathrm{SiO}_{2} / \mathrm{Si}$ heterostructure, which harvests the carriers more efficiently than the $\mathrm{Gr} / \mathrm{Si}$ heterostructure. And the device showed a higher responsivity of $635 \mathrm{~mA} / \mathrm{W}$ compared with previous work, owing to the highly effective collection of charge carriers photogenerated in $\mathrm{Si}$ under the $\mathrm{Gr} / \mathrm{SiO}_{2} / \mathrm{Si}$ parts. In addition, Bartolomeo et al. [8] demonstrated a responsivity of $3 \mathrm{~A} / \mathrm{W}$ under white-light illumination by $\mathrm{Gr} / \mathrm{Si}$-nanotip heterojunction, owing that the nanotip patterning enhances the light absorption and the tip-enhanced electric field improves separation of photocarriers and obtains internal gain due to impact ionization. These studies have shown that the performance of $\mathrm{Gr} / \mathrm{Si}$ device can be improved by constructing microstructure on the interface. As the local microstructure is important for the enhancement of photocurrent, it is necessary to check the contribution from different places. Photocurrent mapping is a powerful tool to map the photocurrent magnitude on the sensitive area, which can provide the detailed photocurrent contribution from difference places.

In this paper, photocurrent mapping was used to study the origin of the high performance of the $\mathrm{Gr} / \mathrm{Si}$ photodetector. The performance depended heavily on the sizes of $\mathrm{Gr} / \mathrm{Si}$ and $\mathrm{Gr} / \mathrm{SiO}_{2} / \mathrm{Si}$ heterojunction. We analyzed the photocurrent characteristics in the $\mathrm{Gr} / \mathrm{Si}$ and $\mathrm{Gr} / \mathrm{SiO}_{2} / \mathrm{Si}$ area by photocurrent mapping that the photocurrent near the $\mathrm{Gr} / \mathrm{Si}$ edge is higher than that in other positions, which is attributed to the highly effective collection of photocarriers generated. The device $(r=7.4 \mu \mathrm{m}, L=6.1 \mu \mathrm{m})$ presented a broadband photoresponse in the range from 420 to $1000 \mathrm{~nm}$ with the peak sensitivity of $52 \mathrm{~A} / \mathrm{W}$ at $780 \mathrm{~nm}$, and fast response speed with rise time of $16 \mu$ s and decay time of $52 \mu \mathrm{s}$.

\section{DEVICE FABRICATION AND CHRAC- TERIZATIONS}

The schematic diagram of device structure is shown in

*cylan@uestc.edu.cn 
Figure 1a, and the fabrication process is as follows. A 285 $\mathrm{nm} \mathrm{SiO} 2 / \mathrm{n}$-type $\mathrm{Si}$ was selected as the substrate. The substrate was first patterned by standard UV-lithography technique and then etched by reactive ion etching method, forming circular holes with radius of $r=5 \mu \mathrm{m}$ (Figure $1 \mathrm{~b}$ ). Next, the treated substrate was buffered oxide etch for 1 minutes to ensure completely removing residual $\mathrm{SiO} 2$. Then, ring shaped Ag electrode (width: d) was deposited by combining photolithography and thermal evaporation techniques. The electrode enclosed area contains both the $\mathrm{SiO} 2 / \mathrm{Si}$ (width: $\mathrm{L}$ ) and $\mathrm{Si}$ (radius: r) regions. At last, monolayer graphene was transferred on the substrate, forming $\mathrm{Gr} / \mathrm{Si}$ heterostructure.

The Gr/Si heterojunction was first characterized by a Raman spectrometer (Andor SR-500i-A-R) using a 532 $\mathrm{nm}$ as the excitation source (Figure 1c). The characteristics peak of $\mathrm{Si}$ is at $520.1 \mathrm{~cm}-1$. The monolayer graphene is confirmed by the ratio of $G$ peak to $2 \mathrm{D}$ peak greater than 2. The Ag electrode and Si were set as drain and source, respectively. The electrical and photoresponse properties of the devices were carried out with a Keithley 2636B as the source and measurement units in a home-made probe station system. All devices were tested at room temperature and ambient atmosphere. A supercontinuum white light laser (SC-PRO, YSL Photonics) with an acousto-optic monochromator (AOTF-PRO, YSL Photonics) was used as the light source for the measurements. For simplification, the size of $\mathrm{Gr} / \mathrm{Si}$ region (r) keeps constant, and we consider the change of sizes of $\mathrm{Gr} / \mathrm{SiO} 2 / \mathrm{Si}$ region (L) and Ag electrode (d).

\section{RESULTS AND ANALYSIS}

The photocurrents and responsivities were strongly depended on the L and d, as shown in Figure 2. With a small $\mathrm{L}$, the change of $\mathrm{d}$ leads to significant change of the photocurrent, which is small for large L. In fact, the photogenerated carriers in $\mathrm{Si}$ outside the Ag electrode ring can diffuse to the Si window and collected by Gr, contributing to the photocurrent. The diffusion length for holes in n-type $\mathrm{Si}$ in our lab is about $80 \mu \mathrm{m}[13,14]$. So if the length of L $+d$ is larger than the diffusion length, the contribution from the $\mathrm{Si}$ outside of the Ag ring can be neglect, which is the reason for the little change of the photocurrent with $d$ for large L. When d keeps constant, with the increase of L, the photocurrents increase obviously. It means that the $\mathrm{Gr} / \mathrm{SiO} 2 / \mathrm{Si}$ region shows remarkable photoresponse $[9,10$, $12,15]$. The origin of the increase of the photocurrent is also caused by the diffusion of photogenerated holes in the $\mathrm{Si}$ below the $\mathrm{SiO} 2$ to the $\mathrm{Si}$ window region. In fact, the photocurrent tends to be saturated for large $\mathrm{L}$ due to the limited diffusion length. The photoresponsivity is the ratio of photocurrent to area of photosensitive region. Supposing the contribution from outside the Ag electrode can be neglected, the photosensitive region contains both the $\mathrm{Gr} / \mathrm{SiO} 2 / \mathrm{Si}$ region and $\mathrm{Gr} / \mathrm{Si}$ region, namely, $\mathrm{L}+\mathrm{r}$. With the increase of $\mathrm{L}$, the responsivities decrease. A Gr/Si device with smaller $\mathrm{L}$ shows higher responsivity, and similar findings have been demonstrated in our earlier work [13]. (a)

(b)


Figure 1. (a) Schematic diagram of $\mathrm{Gr} / \mathrm{Si}$ heterostructure device. (b) Schematic diagram of size of $\mathrm{Gr} / \mathrm{Si}, \mathrm{Gr} / \mathrm{SiO}_{2} / \mathrm{Si}$ and $\mathrm{Gr} / \mathrm{Ag} / \mathrm{SiO}_{2} / \mathrm{Si}$ regions. (c) Raman spectrum of $\mathrm{Gr} / \mathrm{Si}$ hetero-

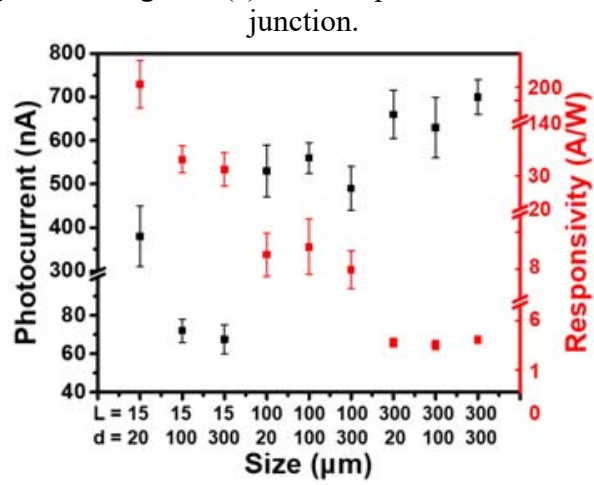

Figure 2 . The photocurrent and responsivity of $\mathrm{Gr} / \mathrm{Si}$ photodetectors with different $L$ and $d$.
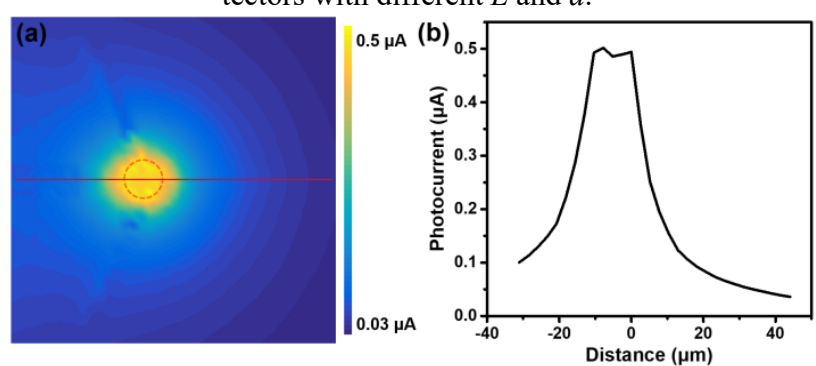

Figure 3. (a) Photocurrent mapping of the device with $r=5 \mu \mathrm{m}$, $L=100 \mu \mathrm{m}$ and $d=100 \mu \mathrm{m}$. (b) The line scan along the red solid line in panel (a).

In order to thoroughly understand the photoresponse of the device with $\mathrm{r}=5 \mu \mathrm{m}, \mathrm{L}=100 \mu \mathrm{m}$ and $\mathrm{d}=100 \mu \mathrm{m}$, photocurrent mapping $(80 \times 80 \mu \mathrm{m} 2)$ was carried out as shown in Figure 3. It is clear that the $\mathrm{Gr} / \mathrm{Si}$ region (red dashed circle) and the $\mathrm{Gr} / \mathrm{SiO} 2 / \mathrm{Si}$ region show photoresponse to incident light (Figure 3a). To get more clear difference of the photocurrent in different positions, a photocurrent profile along the red solid line in Figure 3a was shown in Figure $3 b$. The photocurrent in the $\mathrm{Gr} / \mathrm{Si}$ region is about $0.5 \mu \mathrm{A}$ owing to efficient collection, and drops at the $\mathrm{Si}$ edge. The photocurrent in the $\mathrm{Gr} / \mathrm{SiO} 2 / \mathrm{Si}$ region, originating from carrier diffusion from the illuminated point to the $\mathrm{Gr} / \mathrm{Si}$ junction region driven by electric field $[10,13,16]$, continuously decreases with the increase of radial distance from the Si edge, owing to the increase of photocarriers recombination probability due to the increase of transmission path and defect/impurity in Si. At a distance of $45 \mu \mathrm{m}$ from the edge of $\mathrm{Gr} / \mathrm{Si}$ junction, the photocurrent is only $7 \%$ of the center of $\mathrm{Gr} / \mathrm{Si}$ junction. As a result, to avoid the interference of photocurrent generated outside the electrode, the electrode of device should 
be designed wide enough, which is the reason why the device $(\mathrm{L}=15 \mu \mathrm{m}, \mathrm{d}=20 \mu \mathrm{m})$ in Figure 2 is significantly higher than that $(\mathrm{L}=15 \mu \mathrm{m}, \mathrm{d}=100$ or $300 \mu \mathrm{m})$.

The Schematic band alignment (Figure 4) was performed to understand the working mechanism of the photodetectors, and in particular in the clear photoresponse in the $\mathrm{Gr} / \mathrm{Si}$ and $\mathrm{Gr} / \mathrm{SiO} 2 / \mathrm{Si}$ regions. In the dark state, as shown in Figure 4a, there was a Schottky junction when the semi-metal graphene contacts with n-type $\mathrm{Si}$ to ensure equal Fermi energy, forming a BEF ( $\mathrm{q} \varphi$ ) from Si to Gr and a barrier height of $\mathrm{q} \phi \mathrm{m}$. Under a negative bias $(\mathrm{V})$, as shown in Figure 4b, the Fermi level of Gr will be lift upward due to the finite density of states near the Dirac point, and the band bending of Si near the interface becomes steeper, resulting that the barrier height for electrons from $\mathrm{Gr}$ to $\mathrm{Si}$ is reduced and the BEF increases to $\mathrm{q}(\varphi+\mathrm{V})$. Under light illumination, in the $\mathrm{Gr} / \mathrm{Si}$ region (Figure $4 \mathrm{~b}$ ), electron-hole pairs generated in the bulk $\mathrm{Si}$ will be separated by the BEF. The electrons are transferred to Si side and the holes are transferred towards Gr side, and collected by drain and source electrode, forming photocurrents. In the $\mathrm{Gr} / \mathrm{SiO} 2 / \mathrm{Si}$ region, the Fermi level of $\mathrm{Si}$ in the $\mathrm{Gr} / \mathrm{Si}$ region is lower than that in the $\mathrm{Gr} / \mathrm{SiO} 2 / \mathrm{Si}$ region due to the band bending of $\mathrm{Si}$ in the $\mathrm{Gr} / \mathrm{Si}$ region. The Fermi energy of Si (ESi) can be approximately expressed as white dashed line in Figure 4c. Therefore, an electric field is formed near the edge of $\mathrm{Gr} / \mathrm{Si}$ and $\mathrm{SiO} 2 / \mathrm{Si}$ for $\mathrm{Si}$. The holes photogenerated below the $\mathrm{SiO} 2$ can diffuse laterally to the nearest $\mathrm{Gr} / \mathrm{Si}$ region by the electric field. Red arrows represent the direction of the hole current. With the increase of the distance from the $\mathrm{Gr} / \mathrm{Si}$ edge, the effect of electric field decreases, leading to decrease in the number of holes, resulting in the decrease of photocurrent. Consequently, the photocurrents generation in $\mathrm{Gr} / \mathrm{Si}$ region and $\mathrm{Gr} / \mathrm{SiO} 2 / \mathrm{Si}$ region are significant for the device.

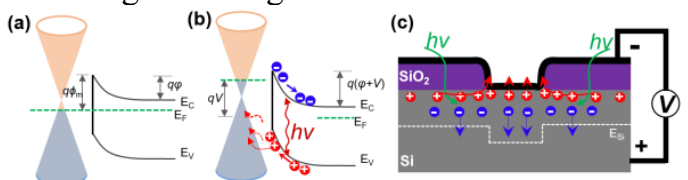

Figure 4. (a) Band diagrams in dark. (b) Band diagrams under reverse bias and light illumination. (c) Schematic of the device under illumination depicting the generation, extraction, and collection of photogenerated charge carriers. $\mathrm{E}_{\mathrm{C}}, \mathrm{E}_{\mathrm{V}}$ and $\mathrm{E}_{\mathrm{F}}$ are the conduction band, valence band and Fermi level, respectively. $\phi_{\mathrm{m}}$ is the barrier height of electron from Gr to Si. $\varphi$ and $\varphi+V$ are the built-in potential barriers after equilibruim and under reverse bias $V$ for $\mathrm{Gr} / \mathrm{Si}$ heterostructure, respectively. Esi is the Fermi level of Si.

To thoroughly understand the photoresponse characterizations, photocurrent mapping $(80 \times 80 \mu \mathrm{m} 2)$ of the device with $\mathrm{r}=12 \mu \mathrm{m}, \mathrm{L}=100 \mu \mathrm{m}$ and $\mathrm{d}=100 \mu \mathrm{m}$ was measured, as shown in Figure 5a. Figure 5b shows the photocurrent profile along the white dashed line in Figure 5a. The device shows the highest photocurrent at the edge of $\mathrm{Gr} / \mathrm{Si}$ and $\mathrm{SiO} 2 / \mathrm{Si}$ junction, which may be due to ballistic transport of photoexcited hot carriers and carrier multiplication gain owing to inhomogeneous boundary induced by wet etching $[9,17]$. The photocurrent in the $\mathrm{Gr} / \mathrm{SiO} 2 / \mathrm{Si}$ continuously decreases with the increasing of distance from the edge. Different from the device with $r=$ $5 \mu \mathrm{m}$, the photocurrents of the device with $\mathrm{r}=12 \mu \mathrm{m}$ in the $\mathrm{Gr} / \mathrm{Si}$ region (red dashed circle) are ununiform, and continuously decrease when the position is near the center. Generally, better photosensitivity near the edge has been observed in the $\mathrm{Gr} / \mathrm{Si}$ heterostructure. Therefore, to obtain high responsivity, the proportion of the area near the edge in the whole photosensitive region should be large enough. Here, with the photocurrent at the center of $\mathrm{Gr} / \mathrm{Si}$ region as reference (blue dashed line in Figure 5b), we obtain the critical value of the optimal size that the radius of $\mathrm{Gr} / \mathrm{Si}$ region (r) is about $7.45 \mu \mathrm{m}$ and the width of $\mathrm{Gr} / \mathrm{SiO} 2 / \mathrm{Si}$ region (L) about $6.15 \mu \mathrm{m}$. In general, a $\mathrm{Gr} / \mathrm{Si}$ photodetector with high responsivity can be demonstrated by controlling size in $r<7.45 \mu \mathrm{m}$ and $\mathrm{L}<6.15 \mu \mathrm{m}$.
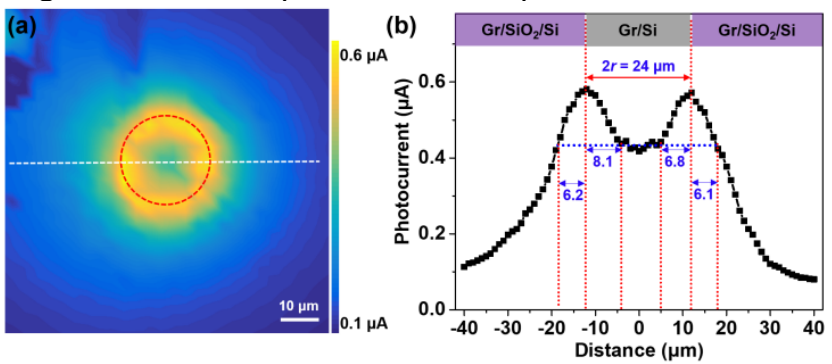

Figure 5. (a) Photocurrent mapping of the device with $r=12$ $\mu \mathrm{m}, L=100 \mu \mathrm{m}$ and $d=100 \mu \mathrm{m}$. (b) The line scan along the white dashed line in panel (a). The diameter ( $2 r)$ of $\mathrm{Gr} / \mathrm{Si}$ region (grey part) is $24 \mu \mathrm{m}$, and the violet part is the $\mathrm{Gr} / \mathrm{SiO}_{2} / \mathrm{Si}$

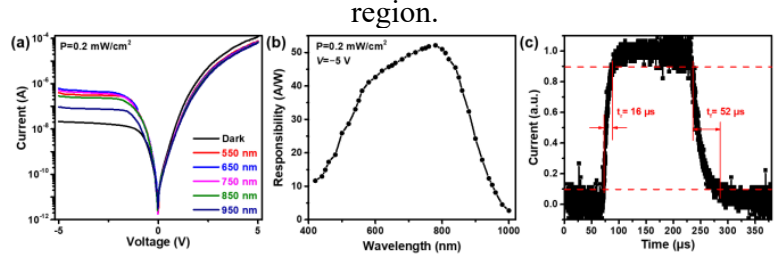

Figure 6. (a) $I-V$ curves characterizations of the device with $r=$ $7.4 \mu \mathrm{m}, L=6.1 \mu \mathrm{m}$ and $d=100 \mu \mathrm{m}$ in dark and under light illumination $\left(0.2 \mathrm{~mW} / \mathrm{cm}^{2}\right)$ with varied wavelengths. (b) Spectral response of the device in the wavelength range of 420-1000 nm under constant light intensity of $0.2 \mathrm{~mW} / \mathrm{cm}^{2}$ at reverse bias -5 V. (c) Time-resolved current under modulated light illumination $\left(\lambda=650 \mathrm{~nm}, P=2.6 \mathrm{~mW} / \mathrm{cm}^{2}\right)$.

We prepared the $\mathrm{Gr} / \mathrm{Si}$ heterostructure photodetector with size of $\mathrm{r}=7.4 \mu \mathrm{m}, \mathrm{L}=6.1 \mu \mathrm{m}$ and $\mathrm{d}=100 \mu \mathrm{m}$, which shows high responsivity. Under different wavelength light illumination $(0.2 \mathrm{~mW} / \mathrm{cm} 2)$, obvious photoresponse can be observed under negative bias voltage, as shown in Figure $6 \mathrm{a}$. To investigate the wavelength-dependent response characteristic, the spectral response was measured as shown in Figure $6 \mathrm{~b}$. It was measured at a fixed incident light intensity of $0.2 \mathrm{~mW} / \mathrm{cm} 2$ in the range of $420-1000$ $\mathrm{nm}$. The photodetector shows high sensitivity and a broadband photoresponse in the range from 420 to $1000 \mathrm{~nm}$ with the peak sensitivity of $52 \mathrm{~A} / \mathrm{W}$ at $780 \mathrm{~nm}$. To determine the response time of the photodetector, high-resolution time-resolved current under modulated light irradiation was measured as shown in Figure 6c. The rise time (tr) and the fall time (tf), defined as the time interval for the current changes from $10 \%$ to $90 \%$ when light is turn on or off, are estimated to be 16 and $52 \mu \mathrm{s}$, respectively. The high photoresponsivity, broadband response, and fast response time indicate the excellent performance of the $\mathrm{Gr} / \mathrm{Si}$ heterostructure photodetector. 


\section{CONCLUSION}

In conclusion, we have demonstrated that the photoresponsivity of $\mathrm{Gr} / \mathrm{Si}$ heterostructure photodetector depends heavily on the sizes of $\mathrm{Gr} / \mathrm{Si}$ region and $\mathrm{Gr} / \mathrm{SiO} 2 / \mathrm{Si}$ region. There is more sensitive photoresponse near the edge of $\mathrm{Gr} / \mathrm{Si}$ and $\mathrm{SiO} 2 / \mathrm{Si}$. With the photoresponse at the center of $\mathrm{Gr} / \mathrm{Si}$ region as reference, the critical values of the optimal size are acquired that the radius of $\mathrm{Gr} / \mathrm{Si}$ region $\mathrm{r}<7.45$ $\mu \mathrm{m}$ and the width of $\mathrm{Gr} / \mathrm{SiO} 2 / \mathrm{Si}$ region $\mathrm{L}<6.15 \mu \mathrm{m}$. And the device with size of $\mathrm{r}=7.4 \mu \mathrm{m}, \mathrm{L}=6.1 \mu \mathrm{m}$ and $\mathrm{d}=100$ $\mu \mathrm{m}$ shows high responsivity of $52 \mathrm{~A} / \mathrm{W}$ at $780 \mathrm{~nm}$. These results provide a strategy for the design of high photoresponsivity $\mathrm{Gr} / \mathrm{Si}$ based devices.

\section{ACKNOWLEDGMENT}

This work is supported by the National Key Research and Development Program of China (No. 2019YFB2203504), Innovation Research Groups of the National Natural Science Foundation of China (Grant No. 61421002), the National Natural Science Foundation of China (Grant Nos. 61605024, 61975024, and 62074024), the Open Fund of Wuhan National Laboratory for Optoelectronics (2018WNLOKF013).

\section{REFERENCES}

1. A. K. Geim; I. V. Grigorieva, "Van der Waals heterostructures," Nature, vol. 499, pp. 419-425, 2013.

2. C. Lan; Z. Shi; R. Cao; C. Li; H. Zhang, "2D materials beyond graphene toward $\mathrm{Si}$ integrated infrared optoelectronic devices," Nanoscale, vol. 12, pp. 1178411807, 2020.

3. W. Tian; H. Sun; L. Chen; P. Wangyang; X. Chen; J. Xiong; L. Li, "Low-dimensional nanomaterial/Si heterostructure-based photodetectors," InfoMat., vol. 1, pp. 140-163, 2019.

4. D. Dragoman; M. Dragoman; R. Plana, "Graphenebased ultrafast diode," J. Appl. Phys., vol. 108, pp. 084316, 2010.

5. T. Wang; D. Huang; Z. Yang; S. Xu; G. He; X. Li; N. Hu; G. Yin; D. He; L. Zhang, "A review on graphenebased gas/vapor sensors with unique properties and potential applications," Nano-Micro Lett., vol. 8, pp. 95-119, 2016.

6. Y. Song; X. Li; C. Mackin; X. Zhang; W. Fang; T. Palacios; H. Zhu; J. Kong, "Role of interfacial oxide in high-efficiency graphene-silicon Schottky barrier solar cells," Nano Lett., vol. 15, pp. 2104-2110, 2015.

7. C. C. Chen; M. Aykol; C. C. Chang; A. F. Levi; S. B. Cronin, "Graphene-silicon Schottky diodes," Nano Lett., vol. 11, pp. 1863-1867, 2011.

8. A. Di Bartolomeo; F. Giubileo; G. Luongo; L. Iemmo; N. Martucciello; G. Niu; M. Fraschke; O. Skibitzki; T. Schroeder; G. Lupina, "Tunable Schottky barrier and high responsivity in graphene/Si-nanotip optoelectronic device," 2D Mater., vol. 4, pp. 015024, 2016.
9. S. Siwapon, "Hybrid graphene-Si-based nanoscale vacuum field effect phototransistors," ACS Photonics, vol. 3, pp. 1799-1808, 2016.

10. S. Riazimehr; S. Kataria; J. M. Gonzalezmedina; S. Wagner; M. Shaygan; S. Suckow; F. G. Ruiz; O. Engstrom; A. Godoy; M. C. Lemme, "High responsivity and quantum efficiency of graphene/silicon photodiodes achieved by interdigitating Schottky and gated regions," ACS Photonics, vol. 6, pp. 107-115, 2019.

11. X. An; F. Liu; Y. J. Jung; S. Kar, "Tunable graphenesilicon heterojunctions for ultrasensitive photodetection," Nano Lett., vol. 13, pp. 909-916, 2013.

12. S. Riazimehr; S. Kataria; R. Bornemann; P. Haring Bolivar; F. J. G. Ruiz; O. Engstrom; A. Godoy; M. C. Lemme, "High photocurrent in gated graphene-silicon hybrid photodiodes," ACS Photonics, vol. 4, pp. 1506-1514, 2017.

13. R. Xiao; C. Lan; Y. Li; C. Zeng; T. He; S. Wang; C. Li; Y. Yin; Y. Liu, "High performance van der Waals graphene-WS $-\mathrm{Si}$ heterostructure photodetector," Adv. Mater. Interfaces, vol. 6, pp. 1901304, 2019.

14. J. A. d. Alamo; R. M. Swanson, "Modelling of minority-carrier transport in heavily doped silicon emitters," Solid-State Electron., vol. 30, pp. 1127-1136, 1987.

15. N. Unsuree; H. Selvi; M. G. Crabb; J. A. Alanis; P. Parkinson; T. J. Echtermeyer, "Visible and infrared photocurrent enhancement in a graphene-silicon Schottky photodetector through surface-states and electric field engineering," 2D Mater., vol. 6, pp. 041004, 2019.

16. B. K. Sarker; E. Cazalas; T. F. Chung; I. Childres; I. Jovanovic; Y. P. Chen, "Position-dependent and millimetre-range photodetection in phototransistors with micrometre-scale graphene on $\mathrm{SiC}, "$ Nat. Nanotechnol., vol. 12, pp. 668-674, 2017.

17. S. Srisonphan; K. Hongesombut, "Tuning the ballistic electron transport of spatial graphene-metal sandwich electrode on a vacuum-silicon-based device," RSC Adv., vol. 5, pp. 2032-2037, 2015. 